\title{
Desigualdades socioeconómicas entre departmentos y su asociación con indicadores de mortalidad en Colombia en $\mathbf{2 0 0 0}$
}

\author{
Jesús Rodríguez García
}

Forma de citar

Rodríguez García J. Desigualdades socioeconómicas entre departamentos y su asociación con indicadores de mortalidad en Colombia en 2000. Rev Panam Salud Publica. 2007; 21(2/3):111-24.

RESUMEN Objetivos. Estudiar las desigualdades de indicadores de mortalidad departamentales con respecto a los valores nacionales colombianos e identificar asociaciones con indicadores socioeconómicos.

Métodos. Los datos del registro de defunciones se ajustaron según la cobertura estimada y se calcularon las tasas y los coeficientes de Gini de mortalidad. Se seleccionaron cinco indicadores socioeconómicos departamentales —el coeficiente de Gini de la distribución de ingresos, el índice de desarrollo humano, el índice del producto interno bruto per cápita, la inversión social per cápita y el porcentaje de la población con cobertura sanitaria pública-y se examinaron las diferencias entre los departamentos y el ámbito nacional. Se calcularon los coeficientes de correlación de Spearman entre los indicadores de mortalidad y los indicadores socioeconómicos seleccionados.

Resultados. La cobertura del registro de defunciones se estimó en un 76\%; el departamento del Chocó presentó el menor valor (35\%) y el de Caldas, el mayor (88\%). El coeficiente de Gini de mortalidad se asoció significativamente con cuatro indicadores socioeconómicos estudiados y la tasa global de mortalidad, con un indicador. La mortalidad por diabetes mellitus se asoció positivamente con todos los indicadores socioeconómicos, las mortalidades por desnutrición y por diarrea, con cuatro indicadores, y la mortalidad por accidentes del tránsito, con dos indicadores. La mortalidad por agresiones no se asoció con ninguno de los indicadores estudiados. Conclusiones. El ajuste de los datos de defunciones permitió obtener indicadores de mortalidad más válidos en la asociación con los indicadores socioeconómicos. El coeficiente de Gini de mortalidad y las mortalidades por desnutrición y diarreas resultaron más idóneos para evaluar las desigualdades interdepartamentales por la mejor asociación presentada con los indicadores socioeconómicos. La asociación encontrada entre la mortalidad por diabetes mellitus y los indicadores socioeconómicos puede deberse a un error sistemático al clasificar dicha enfermedad como causa básica de muerte en departamentos de bajo nivel de desarrollo. El departamento resulta una unidad de análisis muy grande, lo que puede dificultar la identificación de la asociación entre las mortalidades por agresiones y accidentes del tránsito, y los indicadores socioeconómicos.

Palabras clave Equidad en salud, tasa de mortalidad, tablas de vida, factores socioeconómicos, Colombia.

Centro de Proyectos para el Desarrollo (Cendex), Pontificia Universidad Javeriana, Bogotá, Colombia. Toda correspondencia deberá dirigirse a: Jesús
Rodríguez García, Centro de Proyectos para el Desarrollo (Cendex), Pontificia Universidad Javeriana, Carrera Séptima N. ${ }^{\circ} 40-90$, edificio Emilio
Arango, 5. ${ }^{\circ}$ piso, Bogotá, Colombia. Tel.: (57 1) 3208320, extensión 2210. Fax: (57 1) 2889334. Correo electrónico: rodriguez-j@javeriana.edu.co 
El análisis de indicadores de mortalidad para evaluar la situación de salud de una región o un país es muy habitual $\mathrm{y}$, de hecho, proporciona una de las medidas más objetivas que se pueden tener en el campo de la salud.

El análisis de la mortalidad a partir de los registros parroquiales de defunciones, iniciado en Londres a mediados del siglo XVII, permitió el desarrollo progresivo del conocimiento sobre la distribución de las enfermedades en las poblaciones $\mathrm{y}$, consecuentemente, el descubrimiento de sus causas y su prevención. Abraham y David Lilienfeld, en una obra ya clásica (1), comentan detalladamente la importancia de los estudios de la mortalidad en el establecimiento de la epidemiología como disciplina y ciencia. J. Almenara et al. se pronuncian en el mismo sentido en su importante y oportuno trabajo sobre la historia de la bioestadística (2).

Los aportes de William Farr, en el siglo XIX, basados fundamentalmente en el análisis de la mortalidad, son de enorme relevancia $(3,4)$.

Más recientemente, Abdel R. Omran, en sus trabajos sobre las tendencias $\mathrm{y}$ los comportamientos de poblaciones con diversos grados de desarrollo socioeconómico, concibió el concepto de «transición epidemiológica», muy ligado a la observación de las tendencias en la distribución de las muertes por edades y causas $(5,6)$.

Se definen como desigualdades las diferencias sistemáticas y persistentes entre individuos o grupos sociales o territoriales; estas diferencias reciben el calificativo de inequitativas cuando son injustas (7). Por ejemplo, pueden considerarse desigualdades en salud no inequitativas aquéllas que sólo están determinadas por factores biológicos, que no son controlables ni modificables por el hombre.

En Colombia, C.E. Flórez y colaboradores han indagado sobre las desigualdades en salud de variables como la desnutrición crónica, la mortalidad infantil, los esquemas de vacunación y la cobertura sanitaria, entre distintos grupos poblacionales identificados por características como los ingresos y el estrato socioeconómico (8-10).
En el ámbito internacional también se han estudiado las asociaciones entre las desigualdades en salud y los indicadores socioeconómicos (11-14).

Para evaluar las desigualdades en salud se han empleado instrumentos como las curvas de Lorenz y el coeficiente de Gini, como describen Castillo et al. (15). En una publicación reciente, Shkolnikov y colaboradores (16) presentan un uso un poco diferente de estos procedimientos: el cálculo del coeficiente de Gini como una función adicional de la tabla de mortalidad, para evaluar la desigualdad interindividual de los años-persona vividos hasta la muerte.

En el contexto de las tablas de mortalidad, el coeficiente de Gini puede calcularse mediante la fórmula propuesta por Brown en 1994 (15):

$$
\begin{gathered}
\text { Coeficiente de Gini }= \\
\left|1-\sum_{i=0}^{W}\left(x_{i}+x_{i-1}\right) *\left(y_{i}-y_{i-1}\right)\right|
\end{gathered}
$$

donde los subíndices indican las edades desde 0 hasta el último grupo (80 años y más) y « $y_{i} » \mathrm{y}$ « $x_{i} »$ representan las frecuencias relativas acumuladas de años vividos y muertes hasta la edad «i», respectivamente.

La variable objeto de medición —en este caso, los años-persona vividos hasta la muerte- se construye con los cocientes $\left(t_{i} \cdot d_{i}\right) / d_{i}$, donde $" t_{i}$ " corresponde al valor medio de la edad de defunción y «d $d_{i}$ al número de defunciones que ocurren en el intervalo de edades de $(i, i+n)$. Un valor de uno del coeficiente de Gini indica que todos los individuos fallecen antes de cumplir el primer año de vida, excepto uno que muere en el último grupo de edad, infinitamente alejado del primero. Un valor de cero indica que todas las personas fallecen a la misma edad, lo cual traduce la completa igualdad interindividual en los años que se viven hasta la muerte.

El cálculo del coeficiente de Gini como función de la tabla de mortalidad (llamado en adelante "coeficiente de Gini de mortalidad») puede permitir sintetizar, en una nueva escala de valores, las características de la mortalidad en una determinada población.

En Colombia, la recolección obligatoria y rutinaria de datos de defunciones, que dirige y ejecuta el Departamento Administrativo Nacional de Estadísticas (DANE), permite disponer, de manera regular y confiable, de una buena fuente de datos para el análisis de la situación sanitaria del país en los ámbitos nacional y subnacional (departamentos y municipios). La posibilidad de obtener, además, información sobre indicadores socioeconómicos en el ámbito departamental permite examinar los nexos de estos indicadores entre los departamentos, en el contexto de la gran diversidad sociocultural del país.

En el presente trabajo, se describen los resultados de las estimaciones por departamento de un grupo de indicadores de salud (en términos de mortalidad) y un grupo de indicadores socioeconómicos, y se indaga sobre las posibles asociaciones entre las diferencias de estos indicadores respecto al valor nacional. Dada la diversidad de las características departamentales, la identificación de asociaciones entre indicadores de estos dos grupos puede ser de gran utilidad en trabajos de evaluación y seguimiento de políticas de focalización para la colocación de recursos en materia de salud en el ámbito departamental.

\section{MATERIALES Y MÉTODOS}

La base de datos computacional con el registro de defunciones del DANE del año 2000 se sometió a los siguientes procedimientos de corrección antes de construir y analizar los indicadores de interés:

- Los casos sin especificación de la edad $(0,3 \%)$ se distribuyeron proporcionalmente en los grupos de edades conocidas. En los registros con omisión del departamento de residencia $(1,7 \%)$, se imputó como tal el departamento de ocurrencia de la muerte, dado que la equivalencia entre los departamentos de ocurrencia y de residencia es muy 
alta (aproximadamente de un 90\%). Se excluyeron los registros sin especificación del sexo $(0,02 \%)$.

- Las causas básicas de defunción, codificadas según la Clasificación Internacional de Enfermedades, 10. ${ }^{a}$ revisión (CIE-10) (17), se reunieron, para su análisis, en 139 agrupaciones, que se sometieron a un algoritmo de reclasificación para disminuir los posibles errores de declaración de la causa de la muerte. Este procedimiento se empleó en un estudio realizado por el Centro de Proyectos para el Desarrollo (Cendex), de la Pontificia Universidad Javeriana, para el Instituto Mexicano del Seguro Social (18), el cual, a su vez, se basó en trabajos anteriores (19-22).

- Por último, se ajustó el número de muertes según la estimación de la cobertura del registro de defunciones. Se partió de las tablas de mortalidad por quinquenio, edad, sexo y departamento de residencia construidas por el DANE como parte del estudio de proyecciones de población (tablas de mortalidad para las proyecciones de población 19902015). Las tasas quinquenales se hicieron corresponder al año intermedio de cada quinquenio y las tasas correspondientes al año 2000 se estimaron mediante el ajuste de una regresión lineal. Al multiplicar estas tasas por las poblaciones de cada grupo de edad, según las proyecciones realizadas por el DANE para ese año (proyecciones de población 1985-2005), se obtuvieron los números esperados de defunciones. El cociente resultante de dividir el número registrado de defunciones por el número esperado se consideró como una estimación de la cobertura del sistema. Las tasas ajustadas de mortalidad se obtuvieron multiplicando los números registrados de defunciones por el factor de corrección por subregistro (inverso de la cobertura) calculado por edad, sexo y departamento.

A continuación, se detallan los cálculos realizados, tanto a partir de los datos de mortalidad ajustados como de los datos registrados sin corregir.
Se calcularon, para cada departamento, las tasas de mortalidad por edad, sexo y causa, ajustadas por edad mediante estandarización directa, tomando como referencia la estructura etaria de la población de Colombia en el año 2000.

Se construyeron tablas de mortalidad abreviadas (80 años y más como último grupo de edad), a partir de las tasas específicas de mortalidad, por edad, sexo y departamento. Se siguieron para ello las recomendaciones de Vázquez et al. (23).

Para cada departamento, se calcularon el coeficiente de Gini y la curva de Lorenz derivados de la tabla de mortalidad, a fin de evaluar la desigualdad interindividual de los años-persona vividos hasta la muerte.

Los indicadores socioeconómicos departamentales se obtuvieron de varias fuentes. De cálculos nacionales publicados $(24,25)$ se tomaron el coeficiente de Gini de la distribución de ingresos - determinado mediante encuestas de hogares-, el índice de desarrollo humano (IDH) y el producto interno bruto (PIB) per cápita, el cual se transformó en el índice del PIB per cápita según aparece en el cálculo del IDH (26). Los dos últimos indicadores se corrigieron por la desigualdad en la distribución de los ingresos multiplicándolos por el factor (1-coeficiente de Gini). También de documentos nacionales se tomaron los datos sobre el porcentaje de población con cobertura sanitaria, es decir, afiliada al Sistema General de Seguridad Social en Salud (SGSSS) $(27,28)$, y los montos de inversión social departamental per cápita que, como parte del producto interno bruto, integran las ramas de actividad «aguas, alcantarillados y servicios de saneamiento», «enseñanza» $\mathrm{y}$ «salud y otros gastos sociales» (29).

Para cada variable de interés, se examinaron las desigualdades entre los departamentos y el ámbito nacional. Para ello, se calculó en cada caso la diferencia entre el valor departamental y el nacional, y se dividió el resultado obtenido por el valor nacional, lo que permitió normalizar los datos y facilitó la comparación entre departamentos. Para medir las asociaciones entre las diferencias se empleó el coeficiente de correlación no paramétrico de Spearman.

\section{RESULTADOS}

\section{Cobertura del registro de defunciones}

A partir de un total de 187432 defunciones registradas se obtuvo, al aplicar el factor de corrección por subregistro, un número ajustado de defunciones de 247939 . Esta diferencia refleja una cobertura promedio nacional de un 75,6\% para el año 2000 .

Según estas estimaciones, los departamentos de Caldas (88,2\%), Antioquia $(88,2 \%)$ y Valle $(86,3 \%)$ presentan los mejores porcentajes de cobertura del registro de defunciones, mientras que el Amazonas (33,0\%), el Chocó $(35,0 \%)$ y el Vichada $(41,0 \%)$ presentan los valores más bajos. Estos datos evidencian el alto grado de variación de las coberturas departamentales con respecto al valor nacional estimado.

\section{El coeficiente de Gini como medida de desigualdad interindividual en los años-persona vividos}

En el cuadro 1 se presentan los valores del coeficiente de Gini y de la esperanza de vida al nacer correspondientes a cada departamento, calculados en el contexto de las tablas de mortalidad, con datos registrados de mortalidad sin ajustar y datos ajustados por subregistro.

Más que la diferencia encontrada por departamento - mayor, obviamente, en aquéllos con mayor subregistro de defunciones-, llama la atención la ampliación del intervalo de valores posibles cuando se aplica el factor de corrección.

Los valores del coeficiente de Gini obtenidos a partir de datos de mortalidad sin ajustar se hallaron entre $0,08 \mathrm{y}$ 0,14 , mientras que los valores derivados de datos ajustados variaron entre 0,12 y 0,24. La esperanza de vida al nacer calculada a partir de datos sin 
CUADRO 1. Valores del coeficiente de Gini de mortalidad $\left(G_{m}\right)$ y de la esperanza de vida al nacer $\left(e_{0}\right)$ por departamento en Colombia, 2000, obtenidos a partir de los datos de mortalidad registrados por el DANE, sin ajustar y ajustados por subregistro

\begin{tabular}{|c|c|c|c|c|}
\hline \multirow[b]{2}{*}{ Departamento } & \multicolumn{2}{|c|}{ Datos sin ajustar } & \multicolumn{2}{|c|}{$\begin{array}{l}\text { Datos ajustados } \\
\text { por subregistro }\end{array}$} \\
\hline & $e_{0}$ & $G_{m}$ & $\mathrm{e}_{0}$ & $\mathrm{G}_{\mathrm{m}}$ \\
\hline Amazonas & 76,0 & 0,08 & 64,8 & 0,19 \\
\hline Antioquia & 69,4 & 0,14 & 67,7 & 0,17 \\
\hline Arauca & 71,8 & 0,12 & 64,8 & 0,20 \\
\hline Atlántico & 75,7 & 0,08 & 71,4 & 0,13 \\
\hline Bogotá & 73,6 & 0,10 & 71,2 & 0,13 \\
\hline Bolívar & 76,3 & 0,08 & 71,0 & 0,14 \\
\hline Boyacá & 74,4 & 0,09 & 72,3 & 0,13 \\
\hline Caldas & 70,8 & 0,12 & 68,7 & 0,15 \\
\hline Caquetá & 69,9 & 0,14 & 65,0 & 0,20 \\
\hline Casanare & 74,4 & 0,10 & 67,7 & 0,17 \\
\hline Cauca & 73,0 & 0,11 & 66,6 & 0,18 \\
\hline Cesar & 75,1 & 0,09 & 68,3 & 0,17 \\
\hline Chocó & 75,4 & 0,09 & 62,5 & 0,24 \\
\hline Córdoba & 75,4 & 0,09 & 70,4 & 0,15 \\
\hline Cundinamarca & 75,1 & 0,09 & 69,8 & 0,15 \\
\hline Guainía & 74,8 & 0,10 & 64,9 & 0,20 \\
\hline Guaviare & 73,2 & 0,11 & 64,4 & 0,20 \\
\hline Huila & 71,9 & 0,12 & 69,3 & 0,15 \\
\hline La Guajira & 75,9 & 0,09 & 68,9 & 0,16 \\
\hline Magdalena & 76,2 & 0,08 & 70,4 & 0,15 \\
\hline Meta & 70,6 & 0,12 & 66,9 & 0,17 \\
\hline Nariño & 76,5 & 0,08 & 68,3 & 0,17 \\
\hline Norte Santander & 71,1 & 0,12 & 68,5 & 0,16 \\
\hline Putumayo & 73,8 & 0,11 & 64,5 & 0,20 \\
\hline Quindío & 70,6 & 0,12 & 68,0 & 0,16 \\
\hline Risaralda & 70,9 & 0,12 & 68,2 & 0,16 \\
\hline San Andrés & 76,2 & 0,08 & 72,4 & 0,13 \\
\hline Santander & 72,5 & 0,11 & 69,7 & 0,14 \\
\hline Sucre & 75,5 & 0,09 & 71,5 & 0,14 \\
\hline Tolima & 71,4 & 0,12 & 68,7 & 0,15 \\
\hline Valle & 70,7 & 0,12 & 68,6 & 0,16 \\
\hline Vaupés & 73,1 & 0,12 & 64,2 & 0,19 \\
\hline Vichada & 74,8 & 0,10 & 64,6 & 0,20 \\
\hline Total Colombia & 72,8 & 0,11 & 69,4 & 0,15 \\
\hline
\end{tabular}

ajustar estuvo comprendida entre 69,4 y 76,5 años, mientras que utilizando datos ajustados varió entre 62,2 y 72,4 años.

Por otra parte, los departamentos como Caldas, el Quindío y Risaralda, de mucha tradición en la producción y exportación de café de alta calidad, aparecen entre los diez departamentos de mayor coeficiente de Gini de mortalidad y menor esperanza de vida al nacer cuando los cálculos se efectúan a partir de datos de mortalidad sin ajustar, pero su posición relativa mejora claramente al realizar los cálculos luego de aplicar el factor de corrección por subregistro.
Basándose en los datos ajustados de mortalidad, el departamento del Chocó, de escaso desarrollo industrial y eminentemente agrícola, presentó el mayor grado de desigualdad entre individuos en la distribución de los años-persona vividos hasta la muerte, con un coeficiente de Gini de mortalidad igual a 0,24. A partir de los datos registrados sin ajustar, se estimó un valor de desigualdad en la tabla de mortalidad de 0,09 , que correspondió al segundo menor valor entre todos los departamentos, lo cual, evidentemente, refleja muy mal la realidad.

Con un coeficiente de Gini de mortalidad igual a 0,13 después de aplicar el factor de corrección, los departamentos de San Andrés (conformado por las islas caribeñas de San Andrés y Providencia, de gran atractivo turístico), Boyacá (de gran desarrollo agropecuario), el Atlántico (muy urbano, con un importante desarrollo industrial) y Bogotá (distrito capital del país, de gran desarrollo industrial) presentaron los menores valores de desigualdad, lo cual está en concordancia con el desarrollo relativo alcanzado por estos departamentos. Al calcular el indicador a partir de los datos de mortalidad sin ajustar; San Andrés y el Atlántico compartieron el menor valor de desigualdad $(0,08)$ con departamentos de muy bajo desarrollo, como Magdalena, Nariño, el Amazonas y Bolívar.

Para ilustrar cómo se traducen gráficamente los valores del coeficiente de Gini calculados a partir de datos ajustados de mortalidad, se presentan en la figura 1 las curvas de Lorenz obtenidas para dos departamentos extremos en desigualdad: el Atlántico y el Chocó.

En la figura 2, se presentan las distribuciones proporcionales de la mortalidad por edad para estos dos departamentos y para el ámbito nacional, para ilustrar de otra manera las desigualdades calculadas. En el departamento del Chocó, el perfil de mortalidad por edades presenta un peso proporcional de las muertes de menores de cinco años muy alto, con una disminución de la mortalidad en los años siguientes y un pequeño incremento en los últimos años de vida. En el perfil del departamento del Atlántico, se observa que a partir de 45 años se tiende a acumular la mayor proporción de defunciones.

La correlación entre el coeficiente de Gini de mortalidad y la esperanza de vida al nacer resulta, obviamente, muy alta (en ambas fórmulas se incluyen estimaciones del total de añospersona vividos hasta la muerte); sin embargo, el coeficiente de Gini, al ser una medida de dispersión, está mucho más influenciado por los cambios en las distribuciones de las muertes en las edades extremas que la esperanza de vida, que es una medida promedio (16). 
FIGURA 1. Curva de Lorenz y coeficiente de Gini de mortalidad $\left(G_{m}\right)$ calculados a partir de los datos de defunciones registrados por el DANE y ajustados por subregistro en los departamentos del Atlántico y del Chocó, y en todo el país, Colombia, 2000

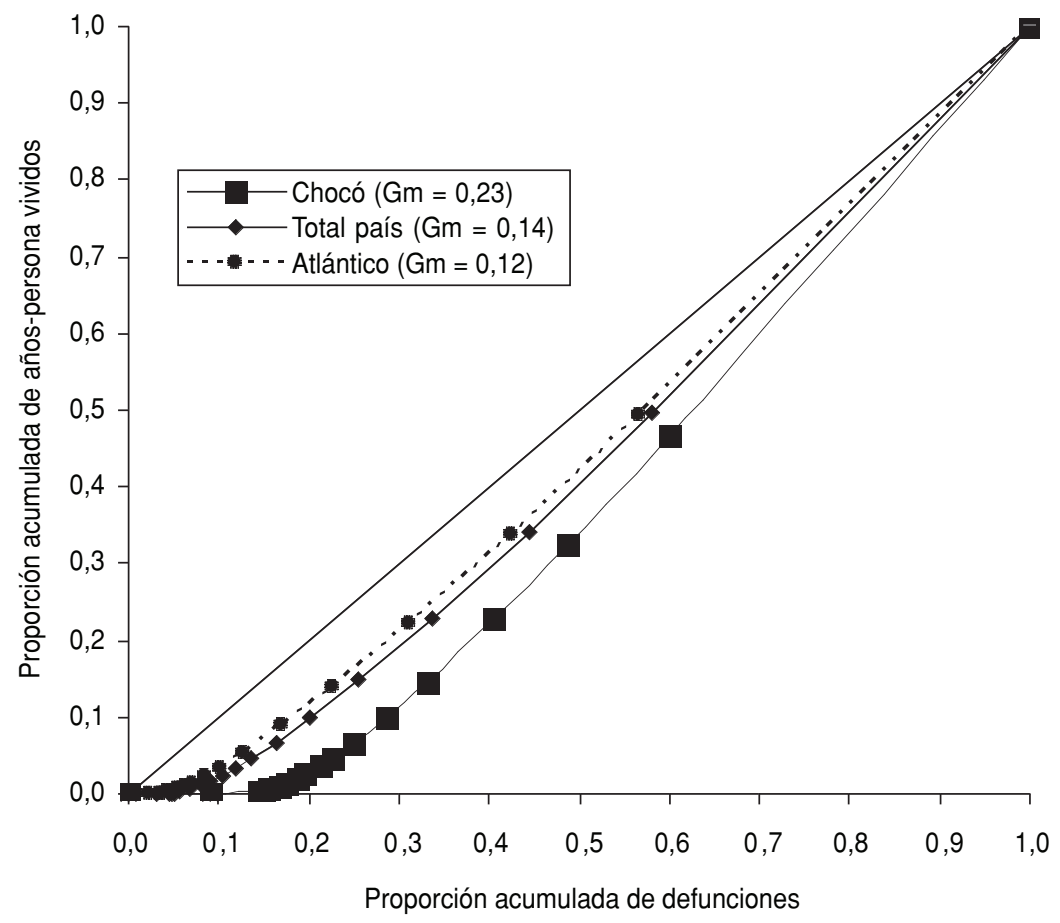

Los resultados obtenidos con datos ajustados de mortalidad, presentados en el cuadro 1 y la figura 2, proporcionan una prueba empírica de lo expre-

sado anteriormente. Por ejemplo, el coeficiente de Gini calculado para el departamento del Atlántico equivale a prácticamente la mitad del valor calcu-

FIGURA 2. Mortalidad relativa por edad, calculada a partir de los datos de defunciones registrados por el DANE y ajustados por subregistro, en los departamentos del Atlántico y del Chocó, y en todo el país, Colombia, 2000

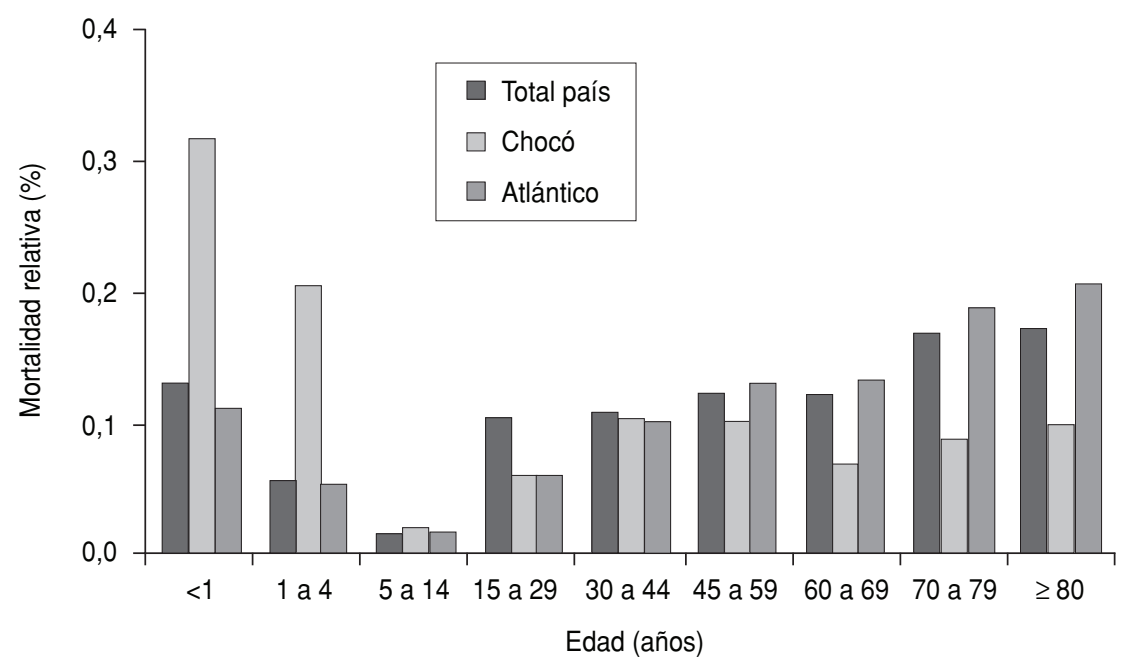

lado para el Chocó (52\%), mientras que la esperanza de vida al nacer de este último departamento representa un $87,5 \%$ de la del Atlántico. Según se observa en la figura 2 , los perfiles de la distribución de la mortalidad son muy distintos en las colas de las distribuciones, es decir, en las edades extremas, lo cual explica la gran diferencia entre los coeficientes de Gini respectivos.

Para situar a Colombia en el contexto internacional de mediciones de desigualdades interindividuales en los años-persona vividos, se revisaron estimaciones de desigualdad entre hombres en algunos países desarrollados y en determinados años, publicadas por V. Shkolnikov et al. (16). Los coeficientes de Gini de mortalidad calculados para los Estados Unidos de América y Suecia en 1900 fueron de 0,37 y 0,33, respectivamente. Los valores determinados para estos dos países en 1995 fueron de 0,13 y 0,10, respectivamente. En 1996, el valor calculado para el Japón fue de 0,11. En 1997, los resultados fueron 0,12 para los Estados Unidos y 0,10 para el Reino Unido. En Colombia, el coeficiente calculado tras el ajuste de los datos de mortalidad fue de 0,18 , es decir, algo mayor que los correspondientes a los Estados Unidos, Japón y el Reino Unido tres años antes. Los valores extremos obtenidos en el ámbito departamental —-0,15 (Boyacá, el Atlántico y Bogotá) y 0,26 (el Chocó)— se hallan dentro del intervalo que puede construirse a partir de las determinaciones para Suecia o los Estados Unidos en los años 1900 y 1995.

\section{Asociación entre los indicadores socioeconómicos y los indicadores de mortalidad por departamento}

En los estudios de la carga de enfermedad se emplea habitualmente, en un primer peldaño de análisis descriptivo, una agregación de causas de mortalidad y morbilidad en tres grandes grupos: enfermedades transmisibles, afecciones perinatales, deficiencias de la nutrición y causas maternas (grupo I) - susceptibles de control con el desarrollo-; enfermedades no transmisibles (grupo II) —cuya frecuencia suele 
aumentar con el desarrollo- y problemas causados por el hombre, es decir, lesiones intencionales y no intencionales (grupo III) —de evolución menos previsible con el desarrollo- (30).

En este estudio se escogieron dos causas de muerte por grupo. Dichas causas se seleccionaron por su importancia entre las principales causas de defunción observadas en el país y por las posibilidades reales de disminuir el riesgo asociado con medidas apropiadas de control. De esta manera, dentro del grupo I se seleccionaron las enfermedades diarreicas (CIE-10: códigos A01-A09) y la desnutrición proteicocalórica (CIE-10: códigos E40-E46), dentro del grupo II, la diabetes mellitus (CIE-10: códigos E10-E14) y la cardiopatía isquémica (CIE-10: códigos I20-I25), y dentro del grupo III, las agresiones (CIE-10: códigos X85-Y09, Y87) y los accidentes del tránsito ${ }^{2}$.

Las variables socioeconómicas escogidas fueron la inversión social per cápita (expresada en dólares corrientes, al cambio oficial a mediados del año 2000), el coeficiente de Gini de la distribución de ingresos, los índices del producto interno bruto per cápita y de desarrollo humano ajustados por la distribución de los ingresos según el coeficiente de Gini y el porcentaje de la población con cobertura sanitaria pública.

En el cuadro 2, se presentan los valores estimados de cada una de las variables incluidas en el análisis de asociación. Para las tasas de mortalidad, se indican entre paréntesis los valores calculados a partir de los datos de defunciones sin ajustar. En este cuadro pueden observarse las diferencias entre las magnitudes de las tasas de mortalidad calculadas antes y después del ajuste por subregistro. Estas diferencias se acentúan para las causas que inciden mayormente en niños menores de cinco años - como las enfermedades diarreicas y la desnutrición proteicocalórica-, entre los cuales suele esti-

\footnotetext{
2 El accidente del tránsito se define como un accidente de transporte que ocurre en la vía pública —calle o carretera-; puede aparecer con códigos entre V01 y V89, con un cuarto dígito indicativo de accidente del tránsito (CIE-10, volumen 1, páginas 962-994).
}

marse el mayor subregistro de defunciones (sobre todo, en los menores de un año).

Se estudió asimismo la asociación entre las diferencias de las variables con respecto a los valores nacionales, dos a dos, mediante el cálculo del coeficiente de correlación de Spearman. En el cuadro 3, se presentan los valores del coeficiente de correlación entre los indicadores de mortalidad, calculados a partir de los datos registrados sin ajustar y los datos ajustados, y los indicadores socioeconómicos. Según puede observarse en este cuadro, la tasa global de mortalidad calculada a partir de datos sin ajustar y estandarizada por edad se asocia de manera significativa y positiva con los indicadores socioeconómicos. El signo del coeficiente de correlación se invierte en todos los casos al calcularlo luego de aplicar el factor de corrección a los datos de defunciones y la significación estadística sólo se mantiene con respecto al índice de desarrollo humano.

El coeficiente de Gini de mortalidad calculado a partir de los datos sin ajustar también presenta correlaciones positivas con los indicadores socioeconómicos. Al aplicar el factor de corrección, el signo del coeficiente de correlación se invierte y la magnitud de la asociación se incrementa considerablemente.

Las tasas de mortalidad por diarrea y desnutrición calculadas a partir de datos sin ajustar presentan correlaciones inversas con los indicadores socioeconómicos, cuyas magnitudes también aumentan de manera importante al aplicar el factor de corrección por subregistro. En cambio, la tasa de mortalidad por cardiopatía isquémica calculada a partir de datos sin ajustar presenta correlaciones directas con las variables socioeconómicas, cuyas magnitudes disminuyen al aplicar el factor de corrección.

Entre los indicadores de mortalidad, la tasa de mortalidad por diabetes mellitus es el único que se asocia significativamente con todos los indicadores socioeconómicos, tanto antes como después de aplicar el factor de corrección por subregistro, alcanzando el valor más alto de correlación frente al índice del producto interno bruto per cápita ajustado. Esta asociación se ilustra bien en la figura 3 .

El coeficiente de Gini de mortalidad y las tasas de mortalidad por diarreas y desnutrición calculados a partir de datos ajustados presentaron asociaciones significativas con cuatro de los cinco indicadores socioeconómicos. En la figura 4 , se presenta gráficamente la asociación entre las diferencias del coeficiente de Gini, calculado a partir de datos ajustados y sin ajustar, y la inversión social per cápita con respecto a los valores nacionales. Se identifica en esta figura la fuerte asociación negativa entre la inversión social y el coeficiente de Gini calculado a partir de datos ajustados de mortalidad.

La asociación positiva importante entre la tasa de mortalidad por enfermedades diarreicas, calculada a partir de datos ajustados o sin ajustar, y el porcentaje de personas afiliadas al SGSSS se logra observar bastante bien en la figura 5 .

La tasa de mortalidad por accidentes del tránsito se asocia de manera significativa y directa con el índice del producto interno bruto per cápita ajustado y el porcentaje de personas afiliadas al SGSSS, con magnitudes un poco superiores cuando los cálculos se realizan a partir de datos sin ajustar.

El indicador de mortalidad menos sensible frente a los indicadores socioeconómicos fue la tasa de mortalidad por agresiones, puesto que su diferencia con respecto al valor nacional no se asoció significativamente con ninguno de ellos, ya sea a partir de datos ajustados o sin ajustar.

Llama la atención que la tasa general de mortalidad ajustada por edad y el coeficiente de Gini de mortalidad presenten entre sí una fuerte correlación directa y exhiban ambos correlaciones positivas significativas y muy similares con la tasa de mortalidad por agresiones, ya sea a partir de datos ajustados o sin ajustar. Sin embargo, de estos indicadores, sólo el coeficiente de Gini de mortalidad es capaz de asociarse de manera importante con todos los indicadores socioeconómicos una vez aplicado el factor de subregistro al número de defunciones, como ya se indicó. 


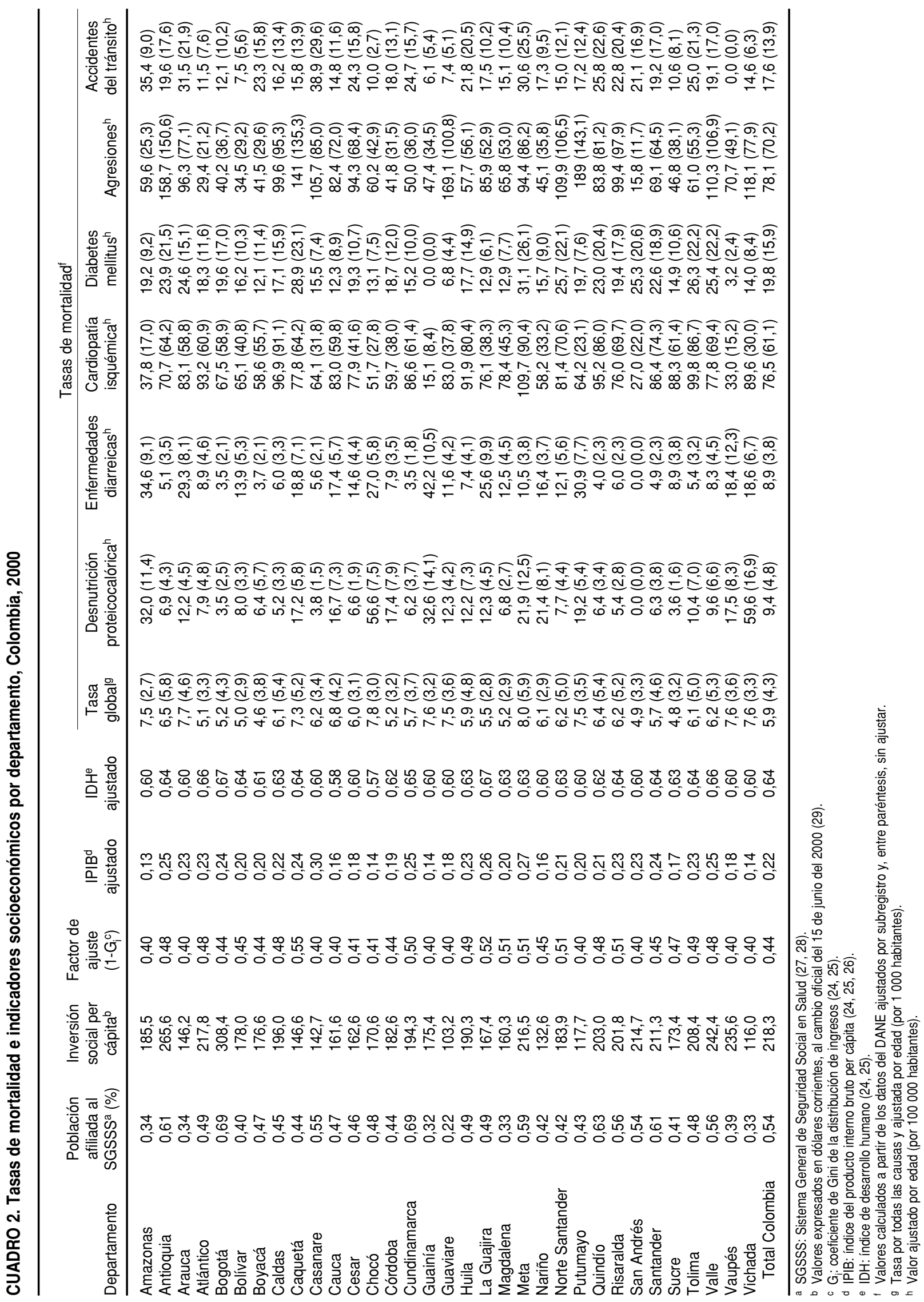


CUADRO 3. Coeficientes de correlación de Spearman entre los indicadores de mortalidad y los indicadores socioeconómicos por departamento, Colombia, 2000

\begin{tabular}{|c|c|c|c|c|c|}
\hline \multirow[b]{2}{*}{$\begin{array}{l}\text { Indicadores socieconómicos } \\
\text { Indicadores de mortalidad }\end{array}$} & \multicolumn{5}{|c|}{ Coeficiente de correlación de Spearmana $(r)$} \\
\hline & $\begin{array}{l}\text { Población afiliada al } \\
\text { SGSSSC }(\%)\end{array}$ & $\begin{array}{l}\text { Inversión social } \\
\text { per cápita }^{d}\end{array}$ & $\begin{array}{l}\text { Factor deajuste } \\
\qquad\left(1-G_{i}^{e}\right)\end{array}$ & $\begin{array}{c}\text { IPIB }^{f} \\
\text { ajustado }\end{array}$ & $\begin{array}{c}\mathrm{IDH}^{\mathrm{g}} \\
\text { ajustado }\end{array}$ \\
\hline Tasa global de mortalidad & $-0,24(0,49)$ & $-0,24(0,45)$ & $-0,29(0,36)$ & $-0,19(0,54)$ & $-0,49(0,31)$ \\
\hline Coeficiente de Gini de mortalidad & $-0,43(0,22)$ & $-0,53(0,24)$ & $-0,36(0,26)$ & $-0,34(0,38)$ & $-0,59(0,16)$ \\
\hline Mortalidad por desnutrición proteicocalórica & $-0,49(-0,31)$ & $-0,35(-0,08)$ & $-0,22(-0,15)$ & $-0,46(-0,36)$ & $-0,41(-0,29)$ \\
\hline Mortalidad por enfermedades diarreicas & $-0,70(-0,64)$ & $-0,57(-0,36)$ & $-0,27(-0,19)$ & $-0,52(-0,42)$ & $-0,45(-0,29)$ \\
\hline Mortalidad por cardiopatía isquémica & $0,22(0,52)$ & $0,10(0,44)$ & $0,47(0,72)$ & $0,30(0,53)$ & $0,36(0,60)$ \\
\hline Mortalidad por diabetes mellitus & $0,43(0,51)$ & $0,40(0,57)$ & $0,36(0,53)$ & $0,52(0,53)$ & $0,36(0,50)$ \\
\hline Mortalidad por agresiones & $-0,07(0,11)$ & $-0,28(-0,13)$ & $0,07(0,23)$ & $0,19(0,32)$ & $-0,09(0,06)$ \\
\hline Mortalidad por accidentes del tránsito & $0,46(0,60)$ & $0,13(0,26)$ & $0,15(0,31)$ & $0,45(0,67)$ & $0,05(0,21)$ \\
\hline
\end{tabular}

${ }^{a}$ Coeficientes calculados a partir de los datos de defunciones ajustados por subregistro y, entre paréntesis, los datos sin ajustar.

$r \geq 0,44$ : correlación significativa, con un nivel de significación de 0,01 (bilateral)

$r \geq 0,35$ : correlación significativa, con un nivel de significación de 0,05 (bilateral)

${ }^{b}$ Indicadores calculados a partir de los datos de defunciones registrados por el DANE.

c SGSSS: Sistema General de Seguridad Social en Salud $(27,28)$.

d (29).

e $\mathrm{G}_{\mathrm{i}}$ : coeficiente de Gini de la distribución de ingresos $(24,25)$.

f IPIB: índice del producto interno bruto per cápita $(24,25,26)$

g IDH: índice de desarrollo humano $(24,25)$.

FIGURA 3. Diferencias entre los valores departamentales y el valor nacional de la tasa de mortalidad (TM) por diabetes mellitus (DM), calculada a partir de los datos de defunciones registrados por el DANE y ajustados por subregistro o sin ajustar, y del índice del producto interno bruto (IPIB) per cápita $(24,25,26)$ ajustado en función del coeficiente de Gini de la distribución de ingresos, Colombia, 2000

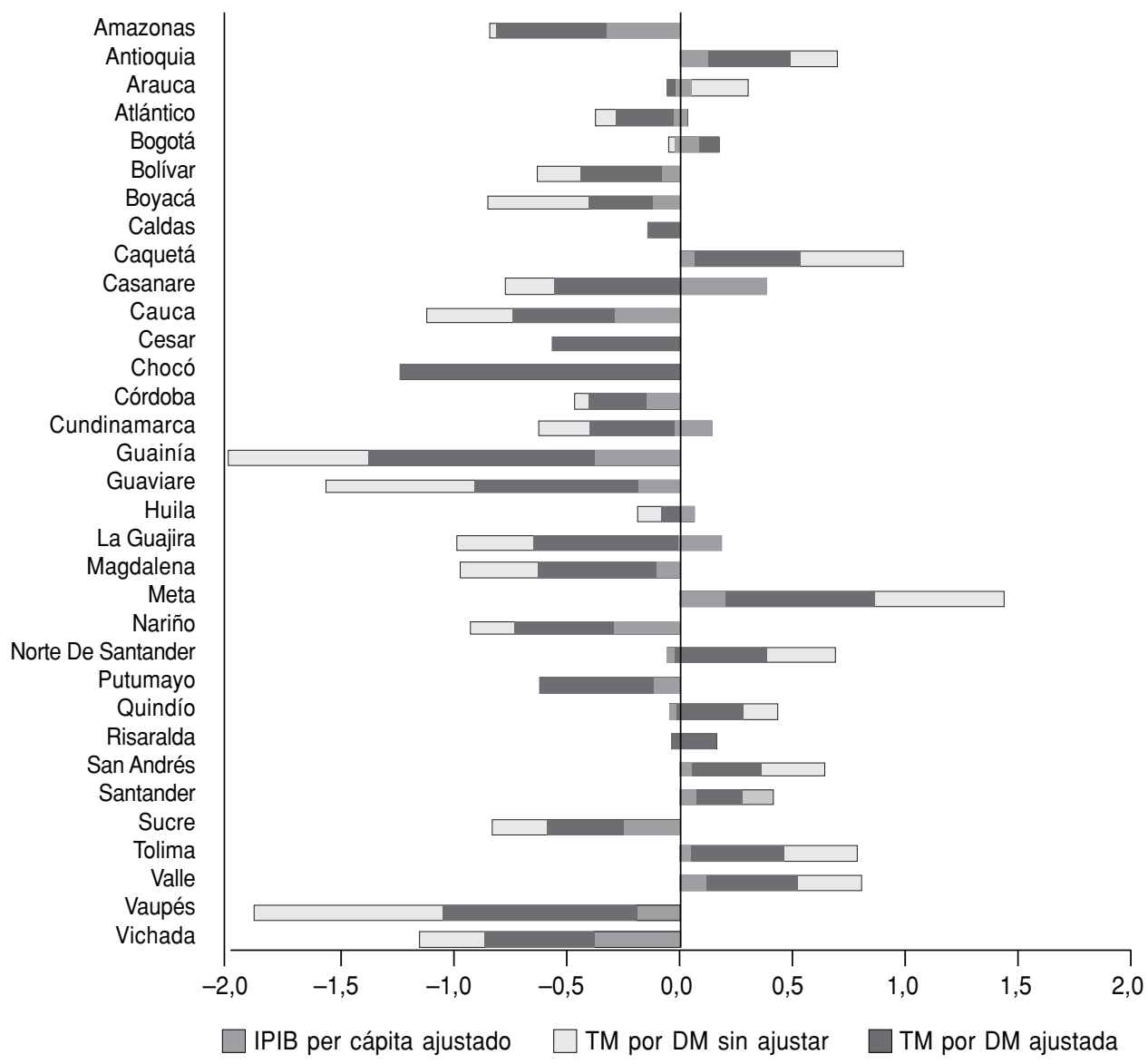




\begin{abstract}
FIGURA 4. Diferencias entre los valores departamentales y el valor nacional del coeficiente de Gini de mortalidad $\left(G_{m}\right)$, calculado a partir de los datos de defunciones registrados por el DANE y ajustados por subregistro o sin ajustar, y del monto en dólares de la inversión social per cápita (29), Colombia, 2000
\end{abstract}

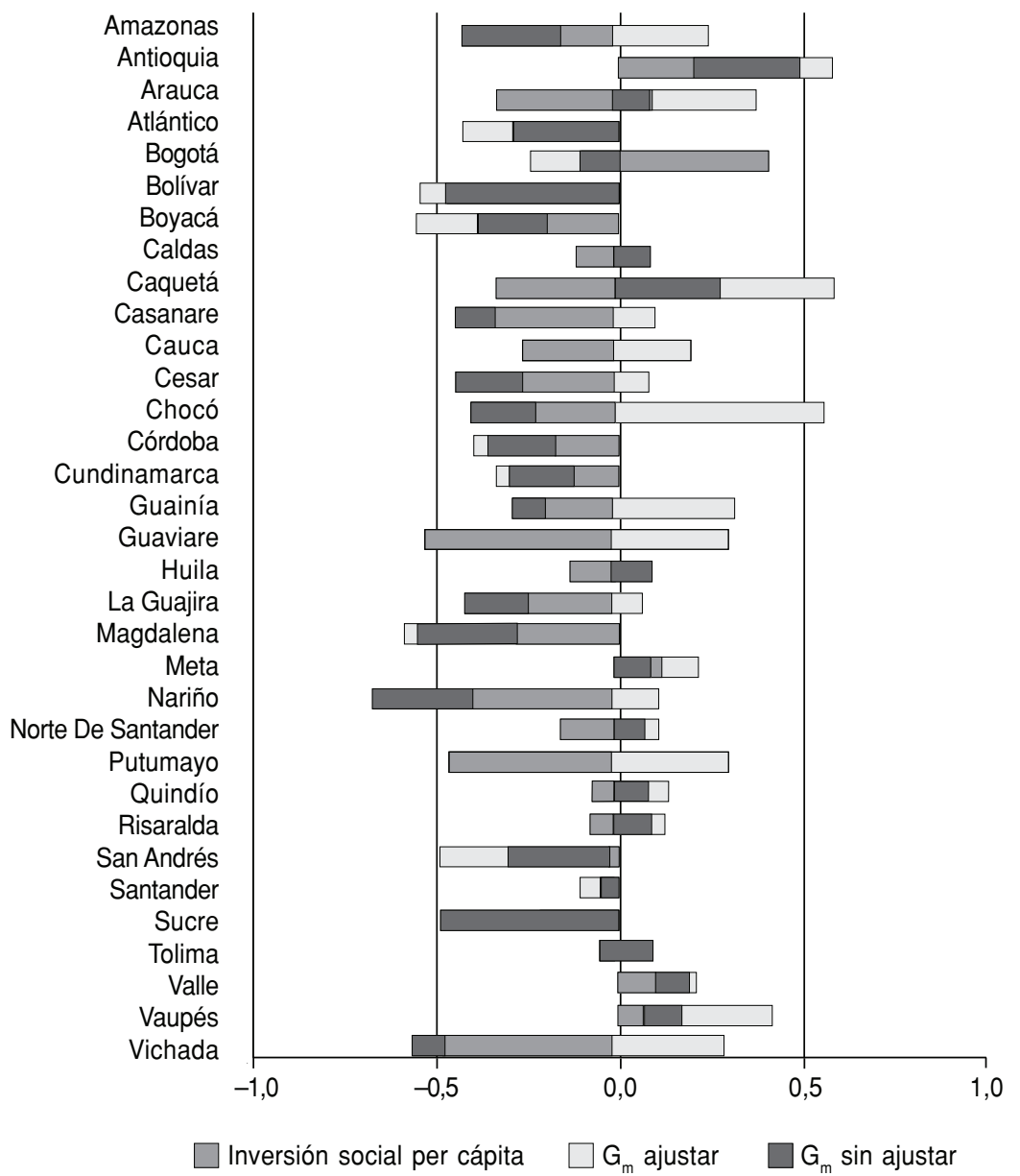

Los indicadores socioeconómicos presentan entre sí una alta correlación de signo positivo. Así, por ejemplo, una mayor inversión social departamental se asocia con una mayor proporción de población con cobertura sanitaria, un mayor producto interno bruto per cápita, un mayor índice de desarrollo humano y una menor desigualdad en el ingreso familiar.

Por último, en el cuadro 4 se presenta, a manera de resumen, la posición de cada departamento en un ordenamiento creciente de desigualdad frente al valor nacional, considerando por separado las sumatorias de las diferencias de los indicadores de mortalidad y de las diferencias de los indicadores socioeconómicos. Las diferencias de los indicadores departamentales de mortalidad con respecto al valor nacional indican una menor desigualdad cuanto más negativas son, mientras que con las diferencias de los indicadores socioeconómicos ocurre lo contrario.

Para el cálculo de la sumatoria de las diferencias de mortalidad se seleccionaron los indicadores que presentaron mayor asociación con los indicadores socioeconómicos, es decir, las tasas de mortalidad por diabetes mellitus, enfermedades diarreicas y desnutrición proteicocalórica, además del coeficiente de Gini de mortalidad. En esta sumatoria, la diferencia de la tasa de mortalidad por diabetes mellitus con respecto al valor nacional se restó de las demás, dada su asociación positiva con los indicadores socioeconómicos.

Para el cálculo de la sumatoria de las diferencias socioeconómicas, se escogieron el índice del producto interno bruto, ajustado por el valor que resulta de restar de la unidad el coeficiente de Gini de la distribución de ingresos, y la inversión social per cápita, por ser indicadores no agregados desde el plano individual, sino globales.

Según se observa en el cuadro 4, el orden de los departamentos determinado por la sumatoria de las diferencias de los indicadores de mortalidad calculados a partir de datos ajustados 


\begin{abstract}
FIGURA 5. Diferencias entre los valores departamentales y el valor nacional de la tasa de mortalidad (TM) por enfermedades diarreicas (ED) ajustada por edad, calculada a partir de los datos de defunciones registrados por el DANE, ajustados por subregistro y sin ajustar, y del porcentaje de personas afiliadas al SGSSS $(27,28)$, Colombia, 2000
\end{abstract}

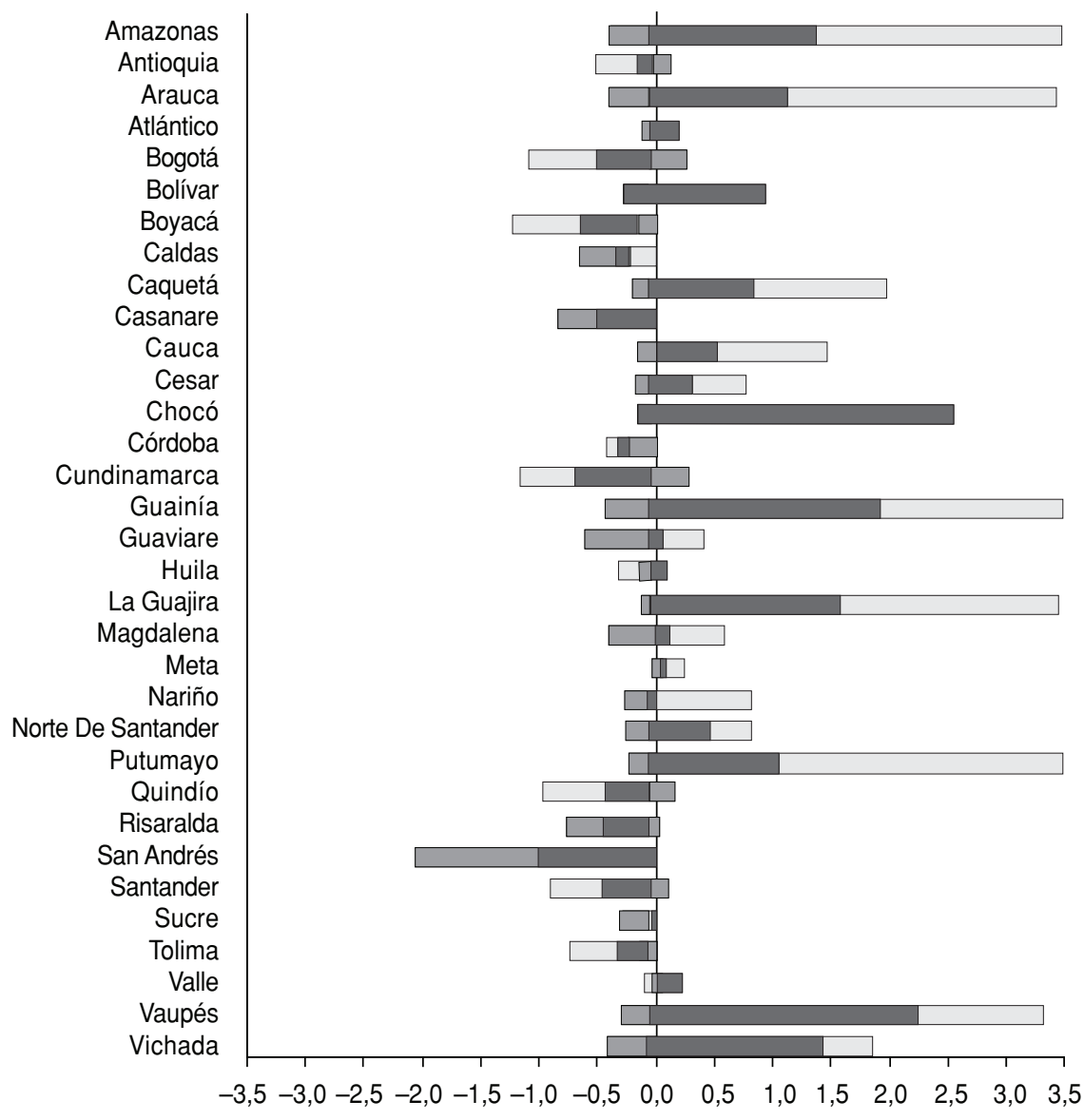

Afiliados al SGSS

TM por ED sin ajustar

TM por ED ajustada

se asemeja mucho más al derivado de la sumatoria de las diferencias de los indicadores socioeconómicos que el obtenido con datos sin ajustar.

\section{DISCUSIÓN}

Aunque resulta imperioso estudiar y evaluar las diferencias de estado de salud entre poblaciones para aportar evidencias que faciliten la mejor toma de decisiones al colocar recursos, no se pueden soslayar las limitaciones de los resultados obtenidos aplicando diseños ecológicos, como en el presente estudio.
En este trabajo, la unidad de análisis es el departamento. La imposibilidad de obtener indicadores socioeconómicos a escala municipal obligó a desechar como unidad de observación este ámbito geográfico, el cual hubiese permitido obtener mayor homogeneidad dentro de las unidades. Se analizaron indicadores de mortalidad agregados desde el ámbito individual e indicadores socioeconómicos, algunos de ellos agregados - como el porcentaje de afiliados al SGSSS y el coeficiente de Gini de la distribución de ingresos- y otros globales, sin medición análoga en el plano individual —como el pro- ducto interno bruto per cápita y la inversión social per cápita.

Desde los trabajos de Morgenstern de 1982 (31), enriquecidos por el propio autor en una publicación más reciente (32), se sabe que las asociaciones establecidas en estudios ecológicos entre características medidas de manera global o agregada (como promedios de mediciones individuales) no reflejan, necesariamente, el grado real de vínculo o relación de estas características en el plano individual.

Sin embargo, dadas las pruebas acumuladas sobre la relación entre el desarrollo socioeconómico y la mortali- 
CUADRO 4. Clasificación de los departamentos según las sumatorias de las diferencias de los indicadores de mortalidad -ajustados por subregistro y sin ajustar-y socioeconómicos con respecto a los valores nacionales, Colombia, 2000

\begin{tabular}{|c|c|c|c|c|c|c|}
\hline \multirow[b]{2}{*}{ Departamento } & \multicolumn{2}{|c|}{ Diferencias de mortalidad } & \multirow[b]{2}{*}{$\begin{array}{c}\text { Diferencias } \\
\text { socioeconómicas }\end{array}$} & \multicolumn{2}{|c|}{$\begin{array}{c}\text { Orden según indicadores } \\
\text { de mortalidad }\end{array}$} & \multirow{2}{*}{$\begin{array}{l}\text { Orden según } \\
\text { indicadores } \\
\text { socioeconómicos }\end{array}$} \\
\hline & $\begin{array}{l}\text { Según datos } \\
\text { sin ajustar }\end{array}$ & $\begin{array}{l}\text { Según datos } \\
\text { ajustados }\end{array}$ & & $\begin{array}{l}\text { Según datos } \\
\text { sin ajustar }\end{array}$ & $\begin{array}{l}\text { Según datos } \\
\text { ajustados }\end{array}$ & \\
\hline Amazonas & 3,14 & 5,56 & $-0,53$ & 18 & 31 & 26 \\
\hline Antioquía & 0,13 & $-0,80$ & 0,36 & 31 & 18 & 2 \\
\hline Arauca & 0,76 & 2,63 & $-0,26$ & 29 & 29 & 20 \\
\hline Atlántico & $-0,59$ & $-0,23$ & 0,04 & 7 & 6 & 8 \\
\hline Bogotá & $-0,64$ & $-1,36$ & 0,52 & 6 & 2 & 1 \\
\hline Bolívar & $-0,34$ & 0,53 & $-0,25$ & 2 & 5 & 19 \\
\hline Boyacá & $-0,70$ & $-0,69$ & $-0,29$ & 12 & 3 & 21 \\
\hline Caldas & $-0,81$ & $-0,65$ & $-0,10$ & 22 & 10 & 14 \\
\hline Caquetá & 1,50 & 1,80 & $-0,24$ & 32 & 26 & 18 \\
\hline Casanare & $-0,59$ & $-0,64$ & 0,04 & 14 & 16 & 7 \\
\hline Cauca & 0,46 & 2,31 & $-0,54$ & 20 & 24 & 27 \\
\hline Cesar & $-0,32$ & 0,45 & $-0,44$ & 11 & 21 & 24 \\
\hline Chocó & 5,33 & 7,95 & $-0,57$ & 8 & 32 & 30 \\
\hline Córdoba & 0,89 & 0,75 & $-0,29$ & 13 & 11 & 22 \\
\hline Cundinamarca & $-0,74$ & $-0,75$ & 0,04 & 5 & 7 & 9 \\
\hline Guainía & 2,57 & 7,53 & $-0,56$ & 19 & 28 & 29 \\
\hline Guaviare & $-0,17$ & 1,57 & $-0,69$ & 10 & 22 & 32 \\
\hline Huila & $-0,01$ & 0,23 & $-0,06$ & 25 & 14 & 13 \\
\hline La Guajira & $-0,09$ & 2,60 & $-0,04$ & 15 & 25 & 12 \\
\hline Magdalena & $-0,76$ & 0,44 & $-0,36$ & 4 & 8 & 23 \\
\hline Meta & 1,81 & 1,06 & 0,22 & 33 & 27 & 4 \\
\hline Nariño & 1,35 & 2,44 & $-0,66$ & 9 & 23 & 31 \\
\hline Norte Santander & 0,10 & $-0,11$ & $-0,21$ & 27 & 20 & 17 \\
\hline Putumayo & 1,47 & 3,82 & $-0,56$ & 23 & 30 & 28 \\
\hline Quindío & $-0,31$ & $-0,98$ & $-0,11$ & 24 & 15 & 16 \\
\hline Risaralda & $-0,38$ & $-0,69$ & $-0,03$ & 21 & 12 & 11 \\
\hline San Andrés & $-0,24$ & $-2,46$ & 0,05 & 1 & 1 & 6 \\
\hline Santander & $-0,34$ & $-0,97$ & 0,06 & 16 & 9 & 5 \\
\hline Sucre & $-1,20$ & $-0,46$ & $-0,44$ & 3 & 4 & 25 \\
\hline Tolima & 0,16 & $-0,61$ & 0,02 & 28 & 17 & 10 \\
\hline Valle & 0,34 & $-0,32$ & 0,25 & 30 & 19 & 3 \\
\hline Vaupés & 0,88 & 3,01 & $-0,10$ & 17 & 13 & 15 \\
\hline Vichada & 5,16 & 7,00 & $-0,83$ & 26 & 33 & 33 \\
\hline
\end{tabular}

dad, estos diseños permiten obtener resultados útiles. Existen desde hace mucho tiempo pruebas suficientes, derivadas de estudios con mediciones individuales, de que las malas condiciones socioeconómicas se asocian con mortalidad prematura. Aunque este hecho ya está aceptado por todos, resulta interesante recordar las palabras del higienista francés Louis René Villermé (1782-1863), cuyos trabajos lo llevaron a afirmar que «la vida del pobre se pierde mucho antes que la del rico» (33).

Por otro lado, también se conoce el efecto que las diferencias en las condiciones socioeconómicas, medidas como un indicador global en un determinado ámbito geográfico, pueden tener en la situación de salud de las personas que habitan en dicho ámbito, según el nivel de ingreso individual $(34,35)$.

Con estos antecedentes, resulta válido esperar una determinada asociación entre indicadores globales o agregados socioeconómicos, e indicadores agregados de mortalidad, en el contexto de un diseño ecológico. Así, en los últimos años se han ensayado, mediante diseños ecológicos, distintas agrupaciones de poblaciones según variables socioeconómicas para evaluar las desigualdades de mortalidad entre los diferentes grupos así formados y su tendencia en el tiempo (36-41).

La unidad territorial departamental resulta evidentemente muy grande, por lo que la información obtenida al agregar los datos a partir de registros individuales, como los de defunciones, puede no ser suficientemente homogénea, lo que puede conducir a que no se exprese la asociación esperada con los indicadores socioeconómicos. Para mitigar esta dificultad, se aplicó a los registros de defunciones un factor de ajuste por subregistro, por edad, sexo y departamento de residencia.

Los resultados obtenidos demostraron las ventajas de ajustar los datos de 
defunciones: se obtuvo mejor coherencia en las asociaciones identificadas entre los indicadores socioeconómicos y los de mortalidad luego de construir estos últimos a partir de los datos de defunciones ajustados por subregistro.

Como antecedente, casi único, de estudio nacional sobre la estimación de la cobertura del registro de mortalidad colombiano por departamentos durante la última década, puede mencionarse un estudio realizado con datos de 1993 por Flórez y Méndez, para el Ministerio de Salud de Colombia ${ }^{3}$. Los factores de ajuste obtenidos en este trabajo se aplicaron a los números de defunciones registrados en dos estudios de análisis de carga de la enfermedad por causa, edad y sexo en Colombia (19) y Bogotá (20).

Aunque existen diferencias metodológicas entre el trabajo de Flórez y Méndez y el presente estudio, la coherencia de algunos resultados hace pertinente la comparación. En los dos trabajos, el orden de clasificación de los departamentos según la magnitud de la cobertura total es muy similar. Así, por ejemplo, el Chocó y Nariño, departamentos con valores de producto interno bruto per cápita entre los más bajos en los años que se comparan (25), presentan coberturas muy bajas.

Las coberturas estimadas para el año 2000 resultaron mayores que las calculadas para 1993 en prácticamente todos los departamentos (incrementos de hasta un 20\%). Esta tendencia también se manifiesta en las estimaciones relativas a Colombia publicadas por la Organización Panamericana de la Salud (OPS), en las que se observa un incremento gradual en la cobertura del registro de defunciones en el ámbito nacional presentada por quinquenios: la estimación para el quinquenio 19901995 fue del 66\% (42), mientras que para el quinquenio 2000-2005 alcanzó el $82,5 \%$ (43).

\footnotetext{
3 Flórez CE y Méndez R. Estimaciones de cobertura del registro de defunciones. Colombia 1993. Informe al Ministerio de Salud, 1997. (Documento fotocopiado).
}

Los resultados obtenidos indican que el coeficiente de Gini permite cuantificar de manera muy sensible la desigualdad en la distribución de la mortalidad por edades: los valores más bajos reflejan una menor desigualdad, con las muertes concentradas en los grupos de mayor edad, mientras que los valores más altos expresan una mayor desigualdad, con la mortalidad más concentrada en los primeros dos grupos de edad. Este indicador presentó una buena asociación con los indicadores socioeconómicos, lo cual lo hace elegible para formar parte de los indicadores de mortalidad utilizados para evaluar desigualdades departamentales según niveles socioeconómicos.

La tasa de mortalidad por diabetes mellitus se asoció de manera directamente proporcional con los indicadores de desarrollo socioeconómico empleados. El ajuste de los datos de mortalidad no modificó esta asociación. Esto parece contradecir lo esperado. Los pacientes diabéticos requieren un tratamiento regular, que incluye una dieta adecuada y ejercicios, así como un seguimiento cuidadoso para vigilar y atender oportunamente las complicaciones que puedan aparecer; en caso contrario, el riesgo de muerte prematura se incrementa considerablemente $(44,45)$. La relación inversa hallada entre la mortalidad y el desarrollo socioeconómico debería verificarse también para la mortalidad por diabetes mellitus.

De hecho, se ha encontrado que en diabéticos, la tasa de mortalidad se incrementa al disminuir el nivel socioeconómico de la población estudiada (46). Aunque en otro estudio no se hallaron diferencias en las tasas de mortalidad por clase socioeconómica entre diabéticos, mientras que se observó una asociación inversa entre la mortalidad y el nivel socioeconómico entre no diabéticos, este resultado podría explicarse por un sesgo de selección de diabéticos en los niveles más bajos (47).

En otro trabajo, se encontró que el nivel socioeconómico de las mujeres con diabetes mellitus era marcada- mente inferior al de las mujeres que no padecían esta enfermedad (48). Entonces, si la frecuencia de la diabetes mellitus es mayor entre las personas de bajo nivel socioeconómico y éstas exhiben tasas de mortalidad más altas, es razonable esperar una relación inversa entre la mortalidad por diabetes mellitus y el nivel socioeconómico.

Por último, cabe la posibilidad de que el resultado de este trabajo esté afectado por un error sistemático en la declaración de la causa básica de muerte si en los departamentos de menor nivel de desarrollo no se estuviese consignando adecuadamente la diabetes mellitus como causa de defunción. Para verificar esta hipótesis es preciso realizar estudios locales que permitan detectar, mediante el cotejo de historias clínicas hospitalarias y certificados de defunción, el subregistro en la declaración de la diabetes mellitus como causa básica de muerte.

Las tasas de mortalidad por diarrea y desnutrición parecen ser indicadores útiles para evaluar las desigualdades entre departamentos de diferentes niveles de desarrollo.

La ausencia de asociación entre la tasa de mortalidad por agresiones y los indicadores socioeconómicos empleados puede ser el resultado del empleo, como unidad de análisis, del departamento, un área geográfica muy grande. La tasa de mortalidad por lesiones resultó asociada de manera creciente con el bajo nivel socioeconómico en un estudio realizado en municipios del Japón (41).

En este estudio se revela la importancia del coeficiente de Gini de mortalidad y las tasas de mortalidad por diarrea y por desnutrición en la identificación de desigualdades entre departamentos de distintos niveles socioeconómicos, así como la ganancia que, en términos de validez, se obtiene cuando se ajustan los datos de defunciones por subregistro. Sin embargo, la selección del ámbito departamental como unidad de análisis para diseños ecológicos de este tipo exige ser cauteloso al emitir conclusiones a partir de resultados obtenidos en este contexto. 


\section{REFERENCIAS}

1. Lilienfeld A, Lilienfeld D. Foundations of epidemiology. Second Edition. Oxford University Press; 1980.

2. Almenara J, Silva LC, Benavides A, García C, González JL. Historia de la bioestadística: la génesis, la normalidad y la crisis. Cádiz: Quorum Editores; 2003. Pp 34-9.

3. Dunn PM. Dr William Farr of Shropshire (1807-1883): obstetric mortality and training. Arch Dis Child Fetal Neonatal Ed. 2002;87(1): F67-9.

4. Eyler JM. Constructing vital statistics: Thomas Rowe Edmonds and William Farr, 1835-1845. Sozpraventivmed 2002;47(1):6-13.

5. Omran AR. The epidemiologic transition: a theory of the epidemiology population change. Milbank Memorial Fund Quarterly. 1971;49(4).

6. Omran AR. The epidemiologic transition in the Americas. Washington, D.C.: Organización Panamericana de la Salud (OPS) y Universidad de Maryland; 1996.

7. Whitehead M. The concepts and principles of equity and health. Int J Health Serv. 1992;22: $430-45$.

8. Flórez CE, Tono TM. La equidad en el sector salud: una mirada de diez años. Bogotá: Fundación Corona y Fundación Ford; 2002 (Documento de trabajo $\mathrm{N} .{ }^{\circ}$ ).

9. Flórez $\mathrm{CE}$, Nupia O. Desnutrición infantil en Colombia: inequidades y determinantes. Documentos CEDE [publicación en línea] 2001. Junio [citada 2 de septiembre de 2004] N. ${ }^{\circ}$ 2001-7:[34 pantallas]. Hallado en: http:// economia.uniandes.edu.co/var/rw/archivos/ cede/documentos/D2001-07.pdf.

10. Flórez CE, Ribero R, Samper B. Health, nutrition, human capital and economic growth in Colombia 1995-2000. Documentos CEDE [publicación en línea] 2003. Noviembre [citada 2 de septiembre de 2004] N. ${ }^{\circ}$. 2003-11: [54 pantallas]. Hallado en: http://economia. uniandes.edu.co/var/rw/archivos/cede/ documentos/d2003-29.pdf

11. Kaplan GA, Pamuk ER, Lynch JW, Cohen RD, Balfour JL. Inequality in income and mortality in the United States: analysis of mortality and potencial pathways. BMJ. 1996;312: 999-1003.

12. Ronzio CR, Pamuk E, Squire GD. The politics of preventable deaths: local spending, income inequality and premature mortality in U.S. cities. J Epidemiol Community Health. 2004; 58:175-9.

13. Kawachi I, Kennedy B, Glass R. Social capital and self-rated health: a contextual analysis. Am J Public Health. 1999;89(8): 1187-93.

14. Kennedy B, Kawachi I, Glass R, ProthrowStith D. Income distribution, socioeconomic status and self-rated health in the United States: multilevel analysis. BMJ. 1998;37: 917-21.

15. Castillo-Salgado C, Schneider C, Loyola E, Mujica O, Roca A, Yerg T. Measuring health inequalities: Gini coefficient and concentration index. Epidemiol Bull. 2001;22(1).

16. Shkolnikov V, Andreev E, Begun A. Gini coefficient as a life table function: computation from discrete data, decomposition of differences and empirical examples. Demographic Res [publicación periódica en línea] 2003. Junio [citada 2 de septiembre de 2004]; 8:[54 pantallas]. Hallado en: http://www. demographic-research.org/volumes/vol8/ 11/8-11.pdf .

17. Organización Panamericana de la Salud (OPS). Clasificación Estadística Internacional de Enfermedades y Problemas Relacionados con la Salud, 10.a revisión, volumen 1 . Wahington D.C.: OPS; 1995. (Publicación científica 554).

18. Rodríguez J, Valdés R, Tao J, Peñalosa RE, Acosta N, O’Meara G. Información depurada de poblaciones, datos de natalidad, morbilidad y mortalidad. México, mayo de 2002. Cendex, Documento técnico ASS/951-02. Hallado en: http://www.cendex.org.co/pdf/ 951-02.pdf. Acceso el 8 de enero de 2005.

19. Rodríguez J, Gallardo H. Carga Global de Enfermedad. Colombia 1985-1995. Bogotá: Ministerio de Salud, Imprenta Nacional; 2000.

20. Gallardo HM, Rodríguez J. La carga de la enfermedad en Santa Fe de Bogotá. Indicadores de años de vida ajustados por discapacidad y mortalidad, 1985-1996. Bogotá: Secretaría Distrital de Salud; 1999.

21. Escobar ML, Gallardo HM, Giraldo PG, Londoño JL, Rodríguez J. La carga de la enfermedad en Colombia. Bogotá: Ministerio de Salud, Editorial Carrera Séptima Ltda; 1994.

22. Lozano R, Murray C, Frenk J, Bobadilla JL, Fernández S. El peso de la enfermedad en México: un doble reto. Serie Economía y Salud: documentos para el análisis y la convergencia, N. ${ }^{\circ}$ 3. México, D.F.: Funsalud; 1994.

23. Vazquez E, Camaño F, Silvi J, Roca A. Life tables: a technique to summarize mortality and survival. Epidemiol Bull. 2003; 24(4):6-10.

24. Colombia, Departamento Nacional de Planeación. Diez años de desarrollo humano en Colombia. Bogotá: Gente Nueva Editorial; 2003.

25. Cuentas departamentales-Colombia. Producto interno bruto departamental por habitante a precios corrientes 1990-2002. [Sitio en Internet] Colombia, Departamento Administrativo Nacional de Estadística (DANE). Hallado en: http://www.dane.gov.co/files/ investigaciones/pib/departamentales/PIB PERCAPITA/PIB_percapita_corrientes 1990-2002.xls. Acceso el 2 de septiembre de 2004.

26. Instituto Valenciano de Investigaciones Económicas y Bancaja. El índice de desarrollo humano en España, 1981-2000. Cuadernos de Capital humano [publicación periódica en línea] 2005. Enero; 49:[8 pantallas]. Hallado en: http://www.ivie.es/downloads/docs/ ch/ch49.pdf. Acceso el 8 de enero de 2005.

27. Colombia, Superintendencia Nacional de Salud (Supersalud). Afiliados al régimen sub- sidiado de salud. Diciembre de 2000. Boletín estadístico N. ${ }^{\circ}$ 1. Bogotá: Supersalud; 2000.

28. Colombia, Superintendencia Nacional de Salud (Supersalud). Afiliados al régimen contributivo de salud. Enero de 2001. Boletín estadístico N. ${ }^{\circ} 3$. Bogotá: Supersalud; 2001.

29. Cuentas departamentales-Colombia. Valor agregado por ramas de actividad económica, a precios corrientes, año 2000. [Sitio en Internet] Colombia, Departamento Administrativo Nacional de Estadística (DANE). Hallado en: http://www.dane.gov.co/files/investigaciones/pib/departamentales/VALOR AGREGADO_ant/PIB-VLR-AGREG-DEPTOCORRIENTES-2000.xls. Acceso el 2 de septiembre de 2004.

30. Murray CJ, Lopez A. Global cause of death patterns in 1990. Bull World Health Organ. 1994;72(3).

31. Morgenstern $\mathrm{H}$. Uses of ecologic analysis in epidemiologic research. Am J Public Health. 1982;72(12):1336-44.

32. Morgenstern H. Ecologic studies. In: Rothman $\mathrm{K}$, and Greenland S. Modern Epidemiology. Second edition. Philadelphia: LippincottRaven Publishers; 1998. Pp. 459-80.

33. Almenara J, Silva LC, Benavides A, García C, González JL. Historia de la bioestadística, la génesis, la normalidad y la crisis. Cádiz: Quorum Editores; 2003. Pp 47-51.

34. Humphreys K, Carr-Hill R. Area variations in health outcomes: artefact or ecology. Int J Epidemiol. 1991;20:251-8.

35. MacRae K. Commentary: socioeconomic deprivation and health and the ecological fallacy. BMJ. 1994;309:1478-9.

36. Smith GD, Whitley E, Dorling D, Gunnell D. Area based measures of social and economic circumstances: cause specific mortality patterns depend on the choice of index. J Epidemiol Community Health. 2001;55:149-50.

37. Singh GK, Miller BA, Hankey BF, Feuer EJ. Changing area socioeconomic patterns in U.S. cancer mortality, 1950-1998: Part I-All cancers among men. J Natl Cancer Inst. 2002;94(12): 904-15.

38. Singh GK, Siahpush M. Increasing inequalities in all-cause and cardiovascular mortality among U.S. adults aged 25-64 years by area socioeconomic status, 1969-1998. Int J Epidemiol. 2002;31:600-13.

39. Singh GK. Area deprivation and widening inequalities in U.S. mortality, 1969-1998. Am J Public Health. 2003;93(7):1137-43.

40. Subramanian SV, Delgado I, Jadue L, Vega J, Kawachi I. Income inequality and health: multilevel analysis of Chilean communities. J Epidemiol Community Health. 2003;57:844-8.

41. Fukuda Y, Nakamura K, and Takano T. Cause-specific mortality differences across socioeconomic position of municipalities in Japan, 1973-1977 and 1993-1998: increased importance of injury and suicide in inequality for ages under 75. Int J Epidemiol. 2005;34: 100-109. 
42. Organización Panamericana de la Salud (OPS). Situación de salud en las Américas. Washington D.C.: OPS; 1998;2:184-97.

43. Organización Panamericana de la Salud (OPS). Situación de salud en las Américas. Indicadores Básicos. Washington D.C.: OPS; 2005.

44. Diabetes Control and Complications Trial Research Group. The effect 123 of intensive treatment of diabetes on the development and progression of long-term complications in insulin-dependent diabetes mellitus. N Engl J Med. 1993;329:977-86.
45. Moss SC, Klein R, Klein BEK, Meuer SM. The association of glycemia and cause-specific mortality in a diabetic population. Arch Intern Med. 1994;154:2473-9.

46. Chaturvedi N, Jarret J, Shipley MJ, Fuller JH. Socioeconomic gradient in morbidity and mortality in people with diabetes: cohort study findings from the Whitehall study and the WHO multinational study of vascular disease in diabetes. BMJ. 1998;316:1005.

47. Koskinen SVP, Martelin TP, Valkonen T. Socioeconomic differences in mortality among diabetic people in Finland: five year follow up. BMJ. 1996;313:975-8.

48. Centers for disease control and prevention. Socioeconomic status of women with diabetesUnited States, 2000. JAMA. 2002;287:2496-7.

Manuscrito recibido el 29 de septiembre de 2004. Aceptado para publicación, tras revisión, el 7 de noviembre de 2005.

ABSTRACT Objectives. To study the inequalities in various mortality indicators for the departments of Colombia with respect to national figures, and to identify associations between the departmental mortality indicators and departmental socioeconomic indicators.

\section{Socioeconomic inequality and its association with mortality indicators in the departments of Colombia in 2000}

Methods. To determine mortality rates and the Gini coefficient for mortality for the departments, data from the death registry were adjusted by the estimated registry coverage for each of the departments. Five socioeconomic indicators were selected: Gini coefficient for income distribution, Human Development Index, per capita gross domestic product, per capita social investment (in health care, etc.), and percentage of the population with health care services from the country's social security system. The differences among the departments were then studied and compared to the national averages. The Spearman's rank correlation coefficient was calculated to find associations between the mortality indicators and each of the five socioeconomic indicators. Results. For Colombia overall, death registry coverage was estimated at $76 \%$, with Chocó department having the lowest coverage $(35 \%)$, and Caldas department the highest ( $88 \%)$. The associations between the Gini coefficient for mortality and four of the socioeconomic indicators studied were significant. The national mortality rate was significantly associated with one socioeconomic indicator. Death caused by diabetes mellitus was associated with all the socioeconomic indicators; death caused by undernutrition or by diarrhea, with four socioeconomic indicators; and death from traffic accidents, with two socioeconomic indicators. Homicide was not associated with any of the socioeconomic indicators studied.

Conclusions. Adjusting the death registry data produced mortality indicators that were more valid for drawing associations with socioeconomic indicators. The Gini coefficient of mortality, mortality from undernutrition, and mortality from diarrheal diseases were more suitable indicators for evaluating the inequalities among the departments because of their higher levels of association with the socioeconomic indicators. Regarding diabetes-related mortality, the associations with all the socioeconomic indicators could be due to systematic errors that lesser-developed departments made when the cause of death was being assigned. A department is a large unit for analysis, which can make it difficult to identify associations between socioeconomic indicators and deaths due to homicide or traffic accidents.

Key words Health status indicators, mortality, life tables, socioeconomic factors, population characteristics, Colombia. 